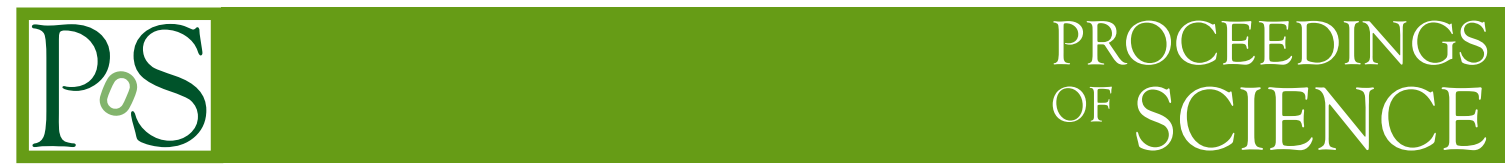

\title{
Status and Prospects for Reactor Experiments
}

\author{
David Lhuillier* \\ CEA, Centre de Saclay, IRFU/Service de Physique Nucléaire \\ F-91191 Gif-sur-Yvette, France. \\ E-mail: david.lhuillierdcea.fr
}

\begin{abstract}
We review the history of reactor neutrino detection, from the pioneer experiments to the modern concept of multi-layered detectors dedicated to the measurement of the last undetermined mixing angle $\theta_{13}$. Experiments heading toward full operations in the next couple of years are presented. Prospects of giant (100's kton) detector as well as growing activity in applied neutrino physics using miniature detectors are briefed.
\end{abstract}

10th International Workshop on Neutrino Factories, Super beams and Beta beams June 30 - July 52008

Valencia, Spain

${ }^{*}$ Speaker. 


\section{Prodigious neutrino sources}

Reactor antineutrino experiments have set up major milestones along the road of neutrino history, starting from the neutrino discovery itself at the Savannah River site [1]. In the late 50's, Reines and Cowan have developed a detection technique that has changed very little since then: a reactor antineutrino interacts with a free proton of a liquid target via inverse $\beta$-decay, producing a positron and a neutron

$$
\bar{v}_{e}+p \rightarrow e^{+}+n
$$

The positron and the resultant annihilation $\gamma$-rays are detected as a prompt signal in liquid scintillator. The neutron forms a delayed signal after thermalization and capture on cadmium. This two-fold coincidence greatly suppresses backgrounds, which is the key of neutrino detection. The kinematics of the reaction implies an energy threshold of $1.8 \mathrm{MeV}$. Above this limit the $\bar{v}_{e}$ energy is accurately inferred from the $e^{+}$energy because of the large mass ratio between $n$ and $e^{+}$. Fissioning heavy isotopes in nuclear reactors produce neutron rich nuclei, which undergo $\beta$-decays when evolving toward the line of stability. Therefore the emitted "neutrino" flux is purely electron antineutrinos. In most reactors the fresh fuel is made of ${ }^{238} U$ enriched to a few percent of ${ }^{235} U$, a fissile isotope. Nevertheless thermalized neutrons can also be captured by ${ }^{238} U$ leading to the production of other fissile isotopes, mainly ${ }^{239} \mathrm{Pu}$ and ${ }^{241} \mathrm{Pu}$. Therefore during a typical fuel cycle the contribution of ${ }^{235} U$ in the total number of fissions decreases while the contributions of $P u$ isotopes grows with time. Since the mean number of antineutrinos per fission differs for each of these isotopes, the above mentioned burnup effect leads to a deviation from the intuitive linearity between the detected flux and the thermal power of the reactor. Therefore single detector experiments have to follow carefully the reactor history to correct for this effect. Lately this sensitivity to the uranium-plutonium content of the core has triggered interest in the international community to use neutrinos as a new safeguard against proliferation of nuclear weapons (see section 6.2).

An important consequence of the energy threshold of inverse beta-decay is that the detected neutrino spectrum is associated with rather large $Q_{\beta}$ transitions meaning short-lived fission products. Therefore the neutrino energy spectrum reaches its equilibrium within few hours, a negligible time with respect to the burnup time scale. Also this dominance of short-lived isotopes above $1.8 \mathrm{MeV}$ prevents a contamination from slower decay of exhausted fuel often stored in the reactor pools.

With a mean energy released by fission of about $200 \mathrm{MeV}$ and mean number of about 2 neutrinos per fission above $1.8 \mathrm{MeV}$, one expects a flux of the order of $10^{20} \bar{v} / \mathrm{s} / G W_{t h}$ in $4 \pi$. This huge flux compensates for the tiny interaction cross-section at low energy and allows reactor neutrino experiments with baseline up to $100 \mathrm{~km}$.

\section{Oscillation Quest}

The few $\mathrm{MeV}$ energy range of nuclear transitions put reactor antineutrinos below the threshold of charged current interactions for muon and tau flavors. Thus evidence of neutrino oscillations at reactors relies on measurement of the survival probability $\mathscr{P}\left(\bar{v}_{e} \rightarrow \bar{v}_{e}\right)$ of the emitted $\bar{v}_{e}$ 's. In the 2 flavors mixing limit the formula writes

$$
1-\mathscr{P}\left(\bar{v}_{e} \rightarrow \bar{v}_{e}\right)=\sin ^{2} 2 \theta \cdot \sin ^{2}\left(1.27 \frac{\Delta m^{2}\left[e V^{2}\right] L[m]}{E_{V}[M e V]}\right)
$$


with $\theta$ the mixing angle, $\Delta m^{2}$ the difference of square masses, $L$ the baseline and $E_{v}$ the antineutrino energy. An asset of reactor experiments is their accurate determination of both $E_{v}$ and $L$, providing a good sensitivity to $\Delta m^{2}$. On the other hand the sought signal of oscillation is to be found in a disappearance of neutrinos with respect to the expected spectrum. It is therefore limited by absolute normalization errors.

In the 80's and early 90's several experiments [2, 3, 4, 5, 6] were performed with few ten's of meters baseline. Typical detection technique of these pioneering experiments was based on detectors segmented in long cells of liquid scintillator, either coupled to ${ }^{3} \mathrm{He}$ counters of loaded with ${ }^{6} \mathrm{Li}$ for neutron detection. Such configuration allowed a good energy resolution and efficient background rejection using the information of location of energy deposition. Main limitations of this approach were a modest detection efficiency (below 50\%), the uncertainty on the free proton number of the fiducial volume, knowledge of the neutrino flux and spectrum shape.

To get rid of the dominant uncertainty of the neutrino source (typical 10\% error on calculated spectra), measurements were taken at several distances from the core using identical detection modules. This method is equivalent to a free normalization of the neutrino source, spoiling the sensitivity to large $\Delta m^{2}\left(\gg E_{v} / L\right)$. In the $\Delta m^{2} \leq E_{v} / L$ domain instead, such relative measurement gets rid of most systematics and can reach high sensitivity in case of large statistics. This approach is actually the basis of modern experiments described in section 5 .

A major improvement in the characterization of the emitted neutrino spectrum were the direct measurements of positron spectra, associated with ${ }^{235} \mathrm{U},{ }^{239} \mathrm{Pu}$ and ${ }^{241} \mathrm{Pu}$ fissions, performed at the ILL research reactor [7]. Subsequent combination with Bugey data [8] pinned down the uncertainty on the integral neutrino rate to $2 \%$ and on the shape distortion to $2 \%$ (bin-to-bin uncorrelated error). Measurement of the main missing contribution of fast neutron induced ${ }^{238} U$ fission is on progress at the FRM2 reactor in Munich.

\section{Atmospheric Parameters}

In the late 90's the Chooz [9] and Palo Verde [10] experiments were motivated by the observation of an anomalous atmospheric neutrino ratio $v_{\mu} / v_{e}$ [11]. The suggested mass parameter was in the range $10^{-2}<\Delta m^{2}<10^{-3} \mathrm{eV}^{2}$, corresponding to an optimal $\simeq 1 \mathrm{~km}$ baseline for a reactor experiment as shown by eq. (2.1).

By it's design the Chooz experiment was a precursor of the next generation of reactor experiments. A one-piece target vessel was filled by 5 tons of Gd-doped liquid scintillator, surrounded by a 70 $\mathrm{cm}$ thick containment region filled with undoped scintillator. Two extra layers provided a muon veto and heavy shielding against rock radioactivity. The use of $\mathrm{Gd}$ brought the dual benefit of reducing the capture time down to few tens of $\mu s$ thanks to its high n-capture cross-section and increasing the energy release in the $\gamma$ cascade following the capture ( $8 \mathrm{MeV}$ compared to 2.2 for hydrogen). The containment region provided a higher detection efficiency $(70 \%)$ as well as a homogeneous detector response. The whole setup was installed in an underground laboratory (300 m.w.e.) providing, for the first time at reactors, a factor 500 reduction of cosmic muon flux from the sea level.

Both Chooz and Palo Verde excluded $\bar{v}_{\mu} \rightarrow \bar{v}_{e}$ oscillation driven by $\Delta m_{\text {atm }}^{2} \leq 10^{-3} \mathrm{eV}^{2}$, except for very small mixing. Assuming CPT this excludes the $v_{\mu} \rightarrow v_{e}$ scenario in the interpretation 
of the atmospheric neutrino results in the SuperKamiokande experiment. As a byproduct, the Chooz result still provides the best constraint on the $\theta_{13}$ mixing angle with $\sin ^{2} 2 \theta_{13}<0.14$ at $\Delta m_{\text {atm }}^{2}=2.510^{-3} \mathrm{eV}^{2}$.

\section{Solar Parameters}

The first reactor antineutrino disappearance was observed by the KamLAND experiment [12] located in Japan, at the site of the earlier Kamiokande experiment, below 2700 m.w.e. of rock. The detector has also a two-layers structure: a 13 m diameter Nylon/EVOH balloon filled with 1 kton of ultra pure liquid scintillator is suspended in a non-scintillating oil buffer contained in an $18 \mathrm{~m}$ diameter stainless steel tank. The KamLAND site is surrounded by 55 commercial nuclear reactors with an average baseline of $180 \mathrm{~km}$. Thus this experiment was sensitive to the Large Mixing Angle oscillation solution of the solar electron neutrino deficit. After a 2881 ton-year exposure the deficit of measured over expected antineutrinos was established at the $8.5 \sigma$ level above 2.6 $\mathrm{MeV}$. An improved analysis including the 2007 data [13] reduced significantly the systematic errors and allowed reaching a $0.9 \mathrm{MeV}$ energy threshold. Then the $L / E$ rate dependence exhibits an impressive pattern of two cycles of oscillation providing strong evidence for neutrino oscillation against other hypothesis such as neutrino decay or decoherence. This measurement provides an unprecedented sensitivity on the solar mass parameter: $\Delta m_{21}^{2}=7.58_{-0.20}^{+0.21} 10^{-5} \mathrm{eV}$.

Detailed analysis of low energy backgrounds provided, for the first time, detection of geo-neutrinos with a direct upper limit of 62 TW (90\% C.L.) for the radiogenic power of U and Th in the Earth.

\section{Chasing $\theta_{13}$}

Among the six parameters related to neutrino oscillation, $\left|\Delta m_{32}^{2}\right|$ and $\sin ^{2} 2 \theta_{23}$ have been measured by atmospheric and long baseline accelerator experiments and $\Delta m_{21}^{2}$ and $\sin ^{2} 2 \theta_{12}$ are known from the combination of solar and KamLAND experiments. $\sin ^{2} 2 \theta_{13}$, the CP violating phase $\delta_{C P}$ and the sign of $\Delta m_{32}^{2}$ are still unknown. However $\theta_{13}$ is already constrained to be much smaller than the two other mixing angles by the negative result of the Chooz experiment (see section 3 ). This parameter translates the admixture of the third mass eigenstate in $v_{e}$ and hence drives the amplitude of the still unobserved $v_{x} \rightarrow v_{y}$ oscillations (where $x$ or $y$ is $e$ ) at the baseline determined by $\left|\Delta m_{31}^{2}\right|$. Beyond the understanding of lepton mixing $\theta_{13}$ is also of fundamental interest to access experimentally CP violation in the leptonic sector since it is proportional to $\sin ^{2} 2 \theta_{13}$.

Superbeam neutrino experiments will exploit the $v_{\mu} \rightarrow v_{e}$ channel using $\simeq 1 \mathrm{GeV}$ neutrinos and long baselines. However the contribution of $\theta_{13}$ to the appearance signal is entangled with $\delta_{C P}$ and the mass hierarchy, degrading the accessible knowledge of $\theta_{13}$ [14]. Thus reactor experiments at 1-2 km baseline, sensitive to the disappearance probability (2.1) driven by $\theta_{13}$ and $\left|\Delta m_{13}^{2}\right|$ only, will provide complementary results. The challenge of this later approach is the accurate measurement of the disappearance of a tiny fraction of $\bar{v}_{e}$, requiring a significant reduction of the absolute normalization errors as well as statistical error with respect to previous experiments like Chooz.

Several sites were proposed in the early 2000's to host new reactor experiments dedicated to $\theta_{13}$. The currently remaining proposals are Double Chooz (France), RENO (Korea), Daya Bay (China) and Angra (Brazil). They are presented below, ordered in increasing target volume corresponding 
to so-called first generation experiments (Double Chooz and RENO) and second generation experiments (Daya Bay and Angra).

Their common strategy of error reduction relies on the use of two (sets of) identical detectors. At a near site, few hundred meters from the core, a first detector monitors the $\bar{v}_{e}$ flux and spectrum shape before the oscillations can induce any distortion. At a far site, between 1 and $2 \mathrm{~km}$ from the core, the second detector searches for a departure from the global solid angle effect, the sought signature of oscillation. This approach cancels out most of the reactor related uncertainties which were the dominant ones in the Chooz experiment (1.9\%). The remaining relative normalization error between the two detectors is expected to be less than $0.6 \%$. The detector concept is based on a 3 concentric cylinders, extended from the two-layers design of Chooz and KamLAND. The inner layer is the neutrino target, filled up with Gadolinium doped liquid scintillator (Gd-LS). Extensive R\&D now allows combining long term stability of the optical properties of Gd-LS as well as large attenuation length suitable for the typical $10 \mathrm{~m}^{3}$ target volume of a detection module. The target is surrounded by a layer of undoped liquid scintillator (LS), called $\gamma$-catcher, to prevent energy leakage due to annihilation and n-capture $\gamma$ rays exiting the target vessel. The outer layer is non-scintillating oil buffer, isolating the active part of the detector from the PMT radioactivity and bringing extra shielding against external neutrons and $\gamma$ rays. These 3 inner layers are separated by two acrylic vessels, proven to be well compatible with LS and transparent to scintillation light traveling from the target and $\gamma$-catcher till the PMT fixed on the buffer tank. External sources of backgrounds are reduced by an hermetic muon veto, a heavy shielding against ambient radioactivity and large enough overburden.

\subsection{Double Chooz}

The Double Chooz experiment [15] locates in Ardennes, France, close to the $2 \times 4.3 G W$ twin reactors of the Chooz nuclear power station. The far site re-uses the underground Chooz laboratory taking advantage of the existing tunnel and pit as well as valuable knowledge of background. The baseline is $1050 \mathrm{~m}$ and overburden is $300 \mathrm{~m}$.w.e. Excavation of a $85 \mathrm{~m}$ air ramp plus a $115 \mathrm{~m}$ tunnel will allow installing the near lab $400 \mathrm{~m}$ away from the cores, underneath a natural hill providing a total overburden of 115 m.w.e. Each site has a single neutrino detector. The target volume is filled with 8.3 ton of $0.1 \%$ Gd loaded liquid scintillator made of $20 \%$ PXE and $80 \%$ dodecane plus fluors. The transmittance in the whole wavelength range of PMTs has been measured to remain perfectly stable over 3 years at room temperature. The thicknesses of the $\gamma$-catcher and buffer are $55 \mathrm{~cm}$ and $105 \mathrm{~cm}$ respectively. Scintillation light generated in the target and gamma-catcher is detected by 390 10-in low background PMT's fixed on the buffer vessel. To minimize detector related systematics, the number of free protons in both targets will be accurately determined by a weighting procedure at the filling. During data taking the detector response is fully characterized using radioactive sources, LEDs and laser beam. Therefore the dominant detector-related systematic error is expected to be kept below $0.6 \%$. The total background rate (accidental+correlated) is estimated to be 1-2 events per day in the far detector, to be compared with a neutrino rate of $\simeq 45 /$ day.

The detector integration in the far lab is in progress and first neutrino data, with the far detector only, are expected in Fall 2009. With the improved detector design the sensitivity on $\sin ^{2} 2 \theta_{13}$ is expected to reach 0.06 at $90 \%$ C.L. in 1.5 years. The analysis of this first phase relies on the prediction of the emitted $\bar{v}_{e}$ spectrum. The above quoted sensitivity assumes a $2 \%$ uncertainty on 
the overall normalization and $2 \%$ on the spectrum shape. A simulation work is on progress in the Double Chooz collaboration to improve the accuracy on the predicted neutrino spectrum [16]. Construction of the near lab and installation of the second detector will go over the next two years. The start of the second phase of the experiment is expected in early 2011 with a sensitivity of $\sin ^{2} 2 \theta_{13}<0.03$ (90\% C.L.) after 3 years running with the two detectors.

\subsection{Reno}

RENO [17] is an experiment to be installed at the Yonggwang nuclear power station. The site has favorable geology with nearby hills providing adequate overburden for far (1380 m baseline, 450 m.w.e.) and near (290 m, 120 m.w.e.) detectors. With a total thermal power of $16.4 \mathrm{GW}$ and 15 tons targets the expected $\bar{v}_{e}$ rate is about 3 times higher than in Double Chooz. However this power is shared among 6 reactors lined up over $1.5 \mathrm{~km}$. Therefore the fraction of neutrino flux coming from each reactor is quite different in the near and far detectors, leading to partial cancellation only of the reactor related systematics [18]. The detector design is similar to the Double Chooz one. The projected sensitivity is 0.02 at $90 \%$ C.L. in 3 years.

The excavation of the horizontal tunnels has started and first data taking is expected in 2010.

\subsection{Daya Bay}

Daya Bay experiment [19] will be the first "second generation experiment" to run in near future. Its goal is to further improve the sensitivity to $\sin ^{2} 2 \theta_{13}$ to better than 0.01 at $90 \%$ C.L. using larger mass target, reduction of backgrounds to ultra low level and control of the relative difference in detector response for all detection modules.

Four reactors of $2.9 \mathrm{GW}$ are distributed on 2 separate sites, Daya Bay and LingAo, $1 \mathrm{~km}$ away from each other. Another two cores, called LingAoII, are expected to commission in 2010 and 2011, increasing the total thermal power from 11.6 GW to $17.4 \mathrm{GW}$. The site has ideal topology with nearby hard granite high hills that can provide efficient protection from cosmic rays. About 3 $\mathrm{km}$ of horizontal tunnels are under construction to connect three experimental halls: the Daya Bay near site (DYB), the LingAo near site (LA), and the far site. DYB and LA sites are $360 \mathrm{~m}$ and 500 $\mathrm{m}$ away from the cores respectively, with similar overburden of $\simeq 400 \mathrm{~m}$.w.e. The far site is $1.9 \mathrm{~km}$ from DYB and $1.6 \mathrm{~km}$ from LA with an overburden of 1200 m.w.e.

A total target mass of 160 tons is splitted into 8 identical antineutrino detectors (ADs), two at each near site and four at the far site. The baseline for the detector-related systematics of each AD is $0.4 \%$. If errors have no correlation between modules this number is expected to scale down with the square root of the number of ADs. All ADs will be filled in an underground Liquid Scintillator Hall, to be excavated, with the same batch of Gd-LS and LS and with a reference tank for precise control of the target mass. After a first 3 years phase ADs will be swapped between near and far sites. The Gd-LS candidate is being tested and no degradation has been found after 1.5 years.

An original aspect of the ADs design is the immersion of the buffer tanks in a big water pool, with at least $2.5 \mathrm{~m}$ water in any direction. The pool provides an efficient shielding against fast neutrons and is equipped of 8-in PMTs to form a muon Cherenkov detector (completed by 4 layers of RPCs covering the top of the pool). The final uncertainty on total background rate is expected to remain below $0.1 \%$. Thanks to the use of reflective panels installed at the bottom and the top of the $\gamma$ catcher only 192 8-in PMTs are instrumented in each buffer tank. 
A surface assembly building and the tunnels are under construction. Installation of the ADs will start in 2009 and data taking with all eight detectors in three halls is expected by december 2010.

\section{Prospects}

Ultimately the concept of near-far measurements will be limited by the error of the relative normalization between the two detectors. Decreasing further this systematic seems to be very challenging. Hence the way to push the sensitivity beyond the barrier of $\sin ^{2} 2 \theta_{13}<0.01$ would be to enter in a regime were one gives up using the main oscillation signal, the disappearance of part of the neutrino flux, to exploit only the shape distortion of the energy spectrum. Such approach removes, on principle, all dependence to any normalization factor which can be let free. The price to pay is the accumulation of a huge number of events in order to measure this "second order" oscillation effect with high enough statistical significance. Then the limiting systematics become the $\sigma_{b i n-t o-b i n}$ errors that is any possible energy dependent difference between the signals in the near and far detectors (backgrounds, biased analysis cuts, ...).

\subsection{Angra}

The Angra proposal [20], located in the neighborhood of Rio de Janeiro, Brazil, aims at exploring this detection strategy in the mid-term future. In the initial design a 500-ton detector is put $1500 \mathrm{~m}$ away from the Angra reactors with 2000 m.w.e. overburden. A (statistical only) precision of $\sin ^{2} 2 \theta_{13}<0.006$ at $90 \%$ C.L. could be reached in 3 years. The project is at present in the phase of conceptual design and sensitivity study. Construction could start in 2013. Meanwhile the Brazilian group has joined the Double Chooz collaboration.

Last year the project of a small very near detector has been approved in the framework of safeguards study, described below.

\subsection{Non proliferation}

Besides the development of giant detectors there is a growing interest in the neutrino community for miniature detectors, aiming at controlling the operation of nuclear reactors via the detection of their neutrino flux [21]. As mentioned in section (1), a nuclear reactor produces the fissile isotope ${ }^{239} \mathrm{Pu}$ when burning its uranium. This plutonium can in principle be separated from the rest of the nuclear fuel to build nuclear weapons. To prevent such illicit activity the United Nations have created the International Atomic Energy Agency (IAEA), in charge of the control of peaceful use of atomic energy. Looking for innovative methods, the IAEA has asked members states to make a feasibility study to determine whether antineutrino detection methods might provide practical safeguards tools vias continuous, non-intrusive and unattended measurements of reactors content. If this method proves to be useful, IAEA can require that any new nuclear power plant to be built has to include an antineutrino monitor.

The challenge of these small neutrino detectors is to keep good detection performances while simplifying the design in view of a possible industrial production. A prototype detector with a $1 \mathrm{~m}^{3}$ Gd-LS target and $3 m \times 3 m$ footprint was operated the last 3 years at the San Onofre power station, California, $25 \mathrm{~m}$ away from a $3.5 \mathrm{GW}$ reactor core. With 459 detected neutrinos per day, the data show clear evidence of sensitivity to the plutonium content with a $10 \%$ decrease of the neutrino 
flux along a reactor cycle [22]. In France the Nucifer project [23] has started this year. It aims at improving the performances of such small Gd-LS with an expected 8 times better statistical accuracy inside the same detector footprint. Complementary activities in the US now focus on the development of neutrino detectors without plastic scintillator [24] and detection of coherent neutrino scattering.

A meeting with expert physicists and IAEA will be held in Vienna end of October 2008 to chart a path to a safeguards deployment.

\section{References}

[1] C.L. Cowan et al, Science 124, (1956) 103.

F. Reines and C.L. Cowan, Phys. Rev. 113, (1959) 273.

[2] H. Kwon et al., Phys. Rev. D24 (1981) 1097.

[3] G. Zacek et al., Phys. Rev. D34 (1986) 2621.

[4] A.F. Afonin et al., Sov. Phys. JETP Lett. 66 (1988) 213.

[5] G.S. Vidyakin et al., JETP Lett. 59 (1994) 390.

[6] B. Achkar et al., Nucl. Phys. B434 (1995) 503.

[7] K. Schreckenbach et al., Phys. Lett. B160 (1985) 325; A. A. Hahn et al., Phys. Lett. B218 (1989) 365.

[8] Y. Declais et al., Phys. Lett. B338 (1994) 383; B. Achkar et al., Phys. Lett. B374 (1996) 243.

[9] M. Apollonio et al., Phys. Lett. B466 (1999) 415.

M. Apollonio et al., Eur. Phys. J. C27 (2003) 331-374.

[10] F. Boehm et al., Phys. Rev. D62 (2000) 072002.

[11] Y. Fukuda et al., Phys. Lett. B335:237-245,1994.

[12] K. Eguchi et al., Phys. Rev. Lett. 90 (2003) 021802.

[13] S. Abe et al., Phys. Rev. Lett. 100 (2008) 221803.

[14] P. Huber et al., Nucl. Phys. B665 (2003) 487.

[15] F. Ardellier et al. (Double-Chooz Collaboration), hep-ex/0405032 (2004); http://doublechooz.in2p3.fr

[16] D. Lhuillier et al., in Proc. INPC 2007, Tokyo, Japan; M. Fallot et al., in Proc. Nuclear Data 2007, Nice, France.

[17] Soo-Bong Kim, AIP Conf. Proc. 981 (2008) 205; J. Phys. Conf. Ser. 120 (2008) 052025.

[18] G. Mention, T. Lasserre, D. Motta, hep-ex/0704.0498.

[19] Xinheng Guo et al. (Daya Bay Collaboration), hep-ex/0701029; http://dayabay.ihep.ac.cn

[20] http://www.e-science.unicamp.br/angra

[21] Applied Antineutrino Physics workshop, Paris (2007), http://www.apc.univ-paris7.fr/AAP2007/

[22] N.S. Bowden et al., Nucl. Instrum. Meth. A572 (2007) 985; A. Bernstein et al., Appl. Phys. 103 (2008) 074905.

[23] A. Porta et al., in Proc. Int. Conf. Physics of Reactors: "Nuclear Power: A Sustainable Ressource" (2008).

[24] S. Dazeley et al., Submitted to Nucl. Instrum. Meth. A, nucl-ex/0808.0219. 